\title{
A high incidence of chronic inflammatory bowel disease in patients with Turner's syndrome
}

\author{
W. H. PRICE \\ From the MRC Clinical and Population Cytogenetics Unit and University Department of Medicine, \\ Western General Hospital, Crewe Road, Edinburgh EH4 $2 \mathrm{XU}$
}

SUMMARY Four new cases of chronic inflammatory bowel disease in a series of 135 adult patients with Turner's syndrome observed over an average period of 11 years are reported. The diagnosis in 2 of the patients was ulcerative colitis; the other 2 had clinical and histological features consistent with a diagnosis of Crohn's disease. The incidence is considerably higher than any reported frequency of new cases of inflammatory bowel disease in the general population. Previously reported cases are described and attention is drawn to the severity of the bowel disorder. An apparent association with a karyotype abnormality which includes a structurally abnormal X chromosome is noted.

Chronic non-specific inflammatory bowel disease has been described in at least 5 patients with gonadal agenesis. Of these, 4 had Turner's syndrome and one had XY gonadal dysgenesis. Williams et al. (1966) described a woman of 40 , previously found to have gonadal dysgenesis and other features of Turner's syndrome, who developed severe ulcerative colitis requiring intensive corticosteroid therapy. Seven years later, at the age of 47 , she had a recurrence for which a colectomy was performed, the resected colon showing the typical features of ulcerative colitis with widespread shallow ulceration and crypt abscesses. The cytogenetic studies showed that the patient was a $45, \mathrm{X} / 46, \mathrm{X}, \mathrm{i}(\mathrm{Xq})$ mosaic. The patient died on the eighth postoperative day. Weinrieb et al. (1976) described 2 patients with Crohn's disease who were also found to have features of Turner's syndrome. One developed symptoms at the age of 17 years and the other at 28 years. The younger patient was successfully treated with corticosteroid therapy, but the other required total colectomy and died after operation of a cardiorespiratory arrest. The cytogenetic diagnosis in both patients was a $45, X / 46, X$, $\mathrm{i}(\mathrm{Xq})$ mosaicism. The fourth patient with Turner's syndrome (Keating et al., 1978) developed symptoms of an acute abdomen at the age of 17 years, and at operation a segment of inflamed and thickened ileum was found which had the typical pathological features of Crohn's disease. The karyotype of this patient was $45, X$. The patient with XY gonadal dysgenesis was described by Audier et al. (1962). She was a 17-yearold girl with a chronic inflammatory enterocolitis,

Received for publication 24 November 1978 initially diagnosed as a tuberculous enteritis, but which failed to respond to anti-tuberculous therapy. At operation she had an inflammatory mass in the right iliac fossa involving the caecum, terminal ileum, and ascending colon. A right hemicolectomy was carried out. The histological features were characteristic of Crohn's disease.

This paper describes 4 further cases of non-specific inflammatory bowel disease in a series of 135 patients with Turner's syndrome observed over an average period of 11 years.

\section{Methods}

The 135 patients have all been notified to the MRC Cytogenetics Registry in Edinburgh since 1959. Each year their general practitioners are asked if the patients are still alive and, if so, whether they have been referred to hospital. Causes of death are checked with the records held by the Registrar General and details of reported referrals are obtained from hospital consultants. The series includes only those patients who have attained the age of 15 years, who have clinical evidence of primary gonadal dysgenesis, and a cytogenetic abnormality with a diagnosis of Turner's syndrome (Table). The years of follow-up have been calculated for each patient from the year of notification to the registry (or from their 15th birthday if they were registered before that age) up to December 1977 or the year of death or emigration.

The 135 patients were observed for a total of 1481 years, an average of 11 years per person (Table). During that time, 4 patients were diagnosed as 63 
Table Karyotype and years of follow-up of 135 patients with Turner's syndrome

\begin{tabular}{|c|c|c|}
\hline Karyotype & $\begin{array}{l}\text { No of } \\
\text { patients }\end{array}$ & $\begin{array}{l}\text { No of } \\
\text { follow-up years }\end{array}$ \\
\hline $\begin{array}{l}\text { 45,X } \\
45, X / 46, X X \\
45, X / 46, X Y \\
46, X, i(X q) \\
45, X / 46, X, i(X q) \\
46, X, i(X q) / 47, X, i(X q), i(X q) \\
46, X, \operatorname{del}(X)(p) \\
45, X / 46, X r(X) \\
45, X / 45, X+r \\
\text { 45,X/46,XmarX } \\
\text { Total }\end{array}$ & $\begin{array}{r}75 \\
2 \\
12 \\
10 \\
16 \\
2 \\
4 \\
4 \\
7 \\
3 \\
135\end{array}$ & $\begin{array}{r}784 \\
35 \\
140 \\
126 \\
201 \\
31 \\
38 \\
40 \\
43 \\
43 \\
1481\end{array}$ \\
\hline
\end{tabular}

suffering from a chronic non-specific inflammatory bowel disorder. Two other patients have suffered from chronic relapsing diarrhoea for which no satisfactory diagnosis has yet been made and they will be reported elsewhere.

\section{Case reports}

CASE 1

This patient was born in 1929, Reg. No $162 / 60$. She was referred to an endocrinology clinic at the age of 18 years because of primary amenorrhoea. Height was $137 \mathrm{~cm}$, weight $36.74 \mathrm{~kg}$, and there were no secondary sexual characteristics. A diagnosis of primary gonadal failure was made and she was treated with oestrogens. In 1960, buccal mucosal cells were found to be chromatin negative. The karyotype analysis of peripheral leucocytes was $45, \mathrm{X}$. Thyroid and gastric antibody tests were negative.

Between 1961 and 1964, she had frequent episodes of central abdominal pain, vomiting, and pyrexia which resolved spontaneously. A barium meal and follow through were negative. In 1964, one of these attacks was more persistent and a laparotomy was carried out. The small intestine was found to be grossly abnormal; there were over 20 circumferential lesions of the lower part of the small bowel and the appearances were those of Crohn's disease. Biopsy of the ileum showed non-specific inflammatory tissue only, but a lymph node contained several epithelioid follicles with giant cells, but no central caseation. They were considered to be sarcoid type follicles, but of a kind sometimes seen in Crohn's disease. The Kveim test was negative; a Mantoux test was also negative at tuberculin dilutions of $1 / 10000$ and $1 / 1000$. She was treated with corticosteroids and her symptoms improved. A chest $x$-ray showed evidence of apparently healed tuberculosis, but after treatment was started specimens of sputum were found to contain tubercle bacilli. The corticosteroids were discontinued and anti-tuberculous chemotherapy was substituted. There has been no recurrence of the gastrointestinal symptoms.

CASE 2

This patient was born in 1944, Reg. No 97/63. Sfe was referred to an endocrinology clinic for primawy amenorrhoea and retarded growth at the age of years. Height was $137 \mathrm{~cm}$, weight $35 \mathrm{~kg}$. Secondafy sexual characteristics were absent. She had bilatefal cubitus valgus, abnormally short fourth and fifth metacarpals in both hands, and an early diastotic murmur at the left sternal edge. Left ventriculgr angiography showed a mild degree of aortic incompetence. Buccal mucosal cells were chroma范 positive and some of the bodies were unusually larges. The chromosome analysis of peripheral leucocytes was $46, \mathrm{X}, \mathrm{i}(\mathrm{Xq})$. Thyroid and gastric antibody tesps were negative. Oestrogen replacement therapy wes given for a few months at the age of 18 years.

윽

From the age of 16 years she had suffered from episodes of diarrhoea lasting 2 to 3 days at a tinde, at first at intervals of several months, but by the age of 26 years every week to 10 days. The stool ws described as loose, sometimes watery, containi $\overrightarrow{b s}$ mucus and traces of blood. At sigmoidoscopy, the mucosa of the sigmoid colon and rectum was finely granular and friable. No biopsy of affected mucosa was obtained. A barium enema showed a loss of normal haustration and superficial ulceration in the transverse and descending colon. A diagnosis ff ulcerative colitis was made and the patient was treated with steroid retention enemas and sulphasa $\vec{a}$ zine. She is maintained symptom-free on sulphäsalazine alone.

\section{CASE 3}

This patient was born in 1947, Reg. No 101/63. She was referred to an endocrinology clinic at the age of $14 \frac{1}{2}$ years because of failure to grow. At the age $\bar{g} f$ 16 years she had not menstruated, there was breast development, and there were no other secondary sexual characteristics. Height was $132 \mathrm{~cm}$, weight $50.9 \mathrm{~kg}$. The nipples were widely spaced and there was shortening of the fifth metacarpals. The buccal mucosal cells were chromatin positive ang some Barr bodies appeared larger than normat. The karyotype of peripheral leucocytes wR $45, X / 46, X i(X q)$. Thyroid and gastric antibody tests were negative.

From the age of 16 years she suffered intermittent abdominal discomfort and diarrhoea and, at the age of 19 years, she was admitted to hospital with $: a$ tentative diagnosis of appendicitis. The symptoms settled without operation, but two weeks later sf was readmitted with a recurrence. At laparotome, there was a suppurative peritonitis and distension $\delta \mathrm{f}$ 
the small bowel, a mass in the transverse colon which could not be dissected from omentum, and a pelvic abscess. Caecostomy was performed and drains were inserted into the region of the colonic mass and into the pelvis. Postoperatively, her condition deteriorated and in spite of resuscitative measures she died the same day. At necropsy, 'the bowel lay in coils matted together by purulent exudate. The mucosa showed patchy haemorrhagic areas throughout its length and the wall appeared slightly thickened. The caecum, ascending, and transverse colon were slightly dilated. An abscess was located at the splenic flexure and dissection revealed a tight stricture of the colon with an anterior perforation. The colon proximal to this was greatly dilated to about $9 \mathrm{~cm}$.' The histological appearances were the same throughout the intestine. 'The mucosa showed severe ulceration, a heavy inflammatory infiltrate of macrophages, lymphocytes, red cells, a moderate number of polymorphs, and plasma cells. There were several narrow ulcers penetrating the muscle coats which appeared hypertrophic and oedematous containing large numbers of inflammatory cells, mainly lymphocytes and macrophages. Numerous micro-abscesses were noted in the muscle coats and there was considerable fibrocellular proliferation throughout the wall.' The lesion was considered to resemble Crohn's disease.

\section{CASE 4}

This patient was born in 1951 , Reg. No $230 / 66$. She was referred to an endocrinology clinic at the age of 15 years because of failure to grow and primary amenorrhoea. Height was $131 \mathrm{~cm}$, weight $34.5 \mathrm{~kg}$. Secondary sexual characteristics were absent. She had a low nuchal hair line, bilateral cubitus valgus, and asymmetrical clavicles. Buccal mucosal cells were chromatin positive. The karyotype of peripheral leucocytes was $46, X, \operatorname{del}(X)(p)$. Thyroid and gastric antibody tests were negative. She was treated with ethinyl oestradiol and later with norethisterone.

At the age of 16 years, bowel actions became frequent and the stools loose in consistency. A diagnosis of ulcerative colitis was made and she was treated initially with sulphasalazine, later with systemic corticosteroids, and she required repeated blood transfusions for anaemia. When, at the age of 18 years, a colonic fistula developed in the right iliac fossa, a proctocolectomy and ileostomy were carried out. The resected colon and rectum showed extensive areas of ulceration, pseudopolyps, and a narrowed segment as the result of scarring. Histology confirmed a diagnosis of ulcerative colitis. There were no histological features of Crohn's disease. For the last 8 years, the ileostomy has functioned well and she has been symptom free.

\section{Discussion}

The average annual incidence rate for new cases of ulcerative colitis and regional enteritis in the Oxford area in the 10-year period 1951 to 1960 (Evans and Acheson, 1965) was 6.6 per 100000 and 8.1 per 100000 women. A very similar incidence was found by Monk et al. (1967) among the white population of Baltimore between 1960 and 1963, and the frequency of new cases of these disorders reported from elsewhere is of the same order. The occurrence of 4 new cases in 135 women with Turner's syndrome during a total of 1481 years at risk is, therefore, more than would be expected. If experienced by all patients with Turner's syndrome, it would amount to an annual rate of approximately 270 per 100000 . Though there are geographical and racial differences in the incidence of non-specific inflammatory bowel disease (Monk et al., 1967), variations of this kind are so small that they are not likely, separately or combined, to account for such a high incidence in Turner's syndrome.

The 135 patients are drawn mainly from Scotland and the north of England. They are not a complete sample of all patients with Turner's syndrome in these regions, but since the 4 patients were registered before they developed symptoms or signs of their bowel disease, there could have been no conscious selection of patients at risk of developing these disorders. Moreover, since the frequency of Turner's syndrome at birth is between 1 and 2 per 10000 , and the mortality in infancy and childhood is high, the 135 patients must be a very high proportion of the survivors in a population of approximately 10 million. It would seem, therefore, that the incidence of 4 cases of chronic non-specific enterocolitis in the 135 patients is significantly greater than expected.

Within the series of 135 patients, the 4 who developed bowel disease were not distinguishable by any physical abnormalities. They had no previous or family history of bowel disease and their dietary habits, medication, occupation, and leisure activities were not noticeably different from others in the series. It is remarkable that 3 of the 4 should be chromatin positive with 46 chromosomes, one of the $\mathrm{X}$ chromosomes being structurally abnormal. In 2 of the 3 , the abnormality was an isochromosome of the long arm of the $\mathrm{X}$, an abnormality which was present in only 26 of the series as a whole. Three of the previously reported cases of ulcerative colitis and Crohn's disease in Turner's syndrome also had an isochromosome of the X (Williams et al., 1966; Weinrieb et al., 1976). It is of interest that there have been several reports of autoimmune thyroiditis in patients with this particular chromosome abnormality (Engel and Forbes, 1961; Sparkes and 
Motulsky, 1963; Grumbach and Morishima, 1964), since, among the causes of Crohn's disease and ulcerative colitis that have been considered, autoimmunity has featured prominently (Broberger and Perlmann, 1959).

In conclusion, the 4 patients described in this paper and those previously reported point to a greatly increased risk of severe non-specific inflammatory bowel disease in patients with Turner's syndrome. The highest risk may be in patients whose abnormal karyotype includes a structurally abnormal $\mathrm{X}$ chromosome. A study of the frequency of antibodies to intestinal mucosa in Turner's syndrome would, therefore, be of interest.

I am indebted to colleagues who have reported patients with Turner's syndrome to the Cytogenetics Registry and annually provided information about them; to Mr A. R. Isaac for operative findings, and Dr J. D. McGregor for histology in patient 1; to Professor J. S. Robson for permission to report patient 3, and to Dr D. Thomson for the necropsy report and histology in this patient; to the staff of the MRC Cytogenetics Unit and to Mrs Frackiewicz and her colleagues in particular for meticulous maintenance of the patient register.

\section{References}

Audier, M., Simonin, R., Payan, H., Stahl, A., Serradimigni, A., Rampal, I., Sommer, D., Gabriel, M., and Delmont, J. (1962). Pseudotumoral ileocolic form of Crohn's disease and gonadal agenesis. Marseille Medicine, $\underset{\mathbb{D}}{\Phi}$, Broberger, M. D., and Perlmann, P. (1959). Autoantibodies in human ulcerative colitis. Journal of Experimental Medicine, 110, 657-674.

Engel, E., and Forbes, A. P. (1961). An abnormal mediựsized metacentric chromosome in a women with primay gonadal failure. Lancet, 2, 1004-1005.

Evans, J. G., and Acheson, E. D. (1965). An epidemiologiçal study of ulcerative colitis and regional enteritis in the Oxford area. Gut, 6, 311-324.

Grumbach, M., and Morishima, A. (1964). X-chromosome abnormalities in gonadal dysgenesis: DNA replication. $8 \mathrm{f}$ structurally abnormal X chromosome: relation to thyrold disease. (Abst.) Journal of Pediatrics, 65, 1987-1088.

Keating, S. P., Ternberg, S. L., and Packman, R. (19 Association of Crohn's disease and Turner's syndrome. Journal of Pediatrics, 92, 160-161.

Monk, M., Mendeloff, A. I., Siegel, C. I., and Lilienfeld, (1967). An epidemiological study of ulcerative colitis amd regional enteritis among adults in Baltimore. Gasinoenterology, 53, 198-210.

Sparkes, R. S., and Motulsky, A. C. (1963). Hashimotg's disease in Turner's syndrome with isochromosome $\mathrm{X}$. Lancet, 1, 947.

Weinrieb, I. S., Fineman, R. M., and Sprio, H. M. (1978). Turner's syndrome and inflammatory bowel disease. England Journal of Medicine, 294, 1221-1222.

Williams, E. D., Engel, E., Taft, P. D., and Forbes, A. (1966). Gonadal dysgenesis and ulcerative colitis. Journato $f$ Medical Genetics, 3, 51-55.

Requests for reprints to $\mathrm{Dr}$ W. H. Price, Mk़ Clinical and Population Cytogenetics Unit, Western General Hospital, Crewe Road, Edinburgh E $\bar{g} 4$ 2XU. 